Revista Búsqueda, Vol. 4 Núm. 19:131-148; 2017. ISSN: 0123-9813

\title{
La esclerosis lateral amiotrófica (ELA): ¿cómo afrontan las y los profesionales sociosanitarios su trabajo con las personas afectadas?
}

\author{
Amiotrophic lateral sclerosis (ALS): How do social and health \\ professionals face their work with people suffering from ALS?
}

\author{
Inmaculada González-Herrera ${ }^{1}$
}

${ }^{1}$ Doctoranda y Magíster en Atención Sociosanitaria a la Dependencia por la Universidad de Valencia, España. Trabajadora Social. Directora C.A.M. Xiquets y Coordinadora Piso Portes Obertes, Valencia, España. Identificador (ID) de autora: http://orcid.org/0000-0002-0491-2141; correo: inma2ts@gmail. com

Recibido: 17-10-2017; Aceptado: 13-12-2017

Y os digo que la vida es realmente oscuridad, salvo allí donde hay entusiasmo. Y todo entusiasmo es ciego, salvo donde hay saber. $Y$ todo saber es vano, salvo donde hay trabajo. Y todo trabajo está vacío salvo donde hay amor. ¿Y qué es trabajar con amor? Es poner, en todo lo que hagáis, un soplo de vuestro espíritu.

Khalil Gibran

\section{Resumen}

La esclerosis lateral amiotrófica (ELA) es una enfermedad neurodegenerativa en la que fallan las neuronas llamadas "motoneuronas", lo que provoca una paralización progresiva de los músculos, que afecta a la movilidad e impide la adecuada deglución, habla y respiración, entre otros síntomas. Además, no tiene cura; solo se pueden paliar sus consecuencias para intentar mejorar la calidad de vida de las personas con que padecen la enfermedad, desde distintos ámbitos sociosanitarios. El presente artículo aborda la percepción de las estrategias de afrontamiento de las y los profesionales que atienden a las personas afectadas, su formación para dicho fin y su satisfacción profesional. Así, a partir de veintidós entrevistas semiestructuradas realizadas a profesionales sociosanitarios de la provincia de Valencia (España), se ha realizado un análisis de contenido cualitativo de la información obtenida. El estudio ha identificado distintas estrategias de afrontamiento que utilizan los profesionales (aceptación, humor y separación, entre otras) y cómo estas varían según la función que los profesionales desempeñan en el tratamiento de la enfermedad. Asimismo, se ha evidenciado que los entrevistados tienen importantes carencias en formación para el afrontamiento, la comunicación con los enfermos y el proceso de duelo.

Palabras clave: esclerosis lateral amiotrófica, ELA, profesionales sociosanitarios, burnout, fatiga por compasión, estrategias de afrontamiento. 


\section{Abstract}

Amyotrophic lateral sclerosis (ALS) is a neurodegenerative disease in which neurons called "motoneurons" fail in their function. This failure causes a progressive paralysis of the muscles responsible for mobility, originating an inadequate swallowing, speaking and breathing, among other symptoms. Further, this disease has no cure and the only thing that can be done is mitigate its consequences in an attempt to improve the quality of life of people who suffer it, from different socio-sanitary fields. This article addresses the perception of the coping strategies of the professionals who care for the people affected, their training in those strategies and their professional satisfaction. Thus, from 22 semistructured interviews conducted with socio-health professionals from the province of Valencia (Spain), a qualitative research of the information obtained has been carried out. The study has identified different coping strategies used by professionals (acceptance, humor and separation, among others) and the way in which these strategies vary according to the role that professionals play in the treatment of the disease. Likewise, it has been shown that the interviewees have significant deficiencies in training for coping, communication with sick people and in the mourning process.

Key words: amyotrophic lateral sclerosis, ALS, socio-health professionals, burnout, compassion or empathy fatigue, coping strategies.

\section{Introducción}

\section{La Esclerosis Lateral Amiotrófica}

El neurólogo francés Charcot (18251893) fue el primer estudioso que habló de la Esclerosis Lateral Amiotrófica (ELA) en 1869. Como se sabe, la ELA es una enfermedad neurológica en la que fallan las neuronas llamadas "motoneuronas", lo que provoca una paralización progresiva de los músculos, que afecta a la movilidad e impide la adecuada deglución, habla y respiración, como se verá más adelante.

Además, la ELA en estos momentos no tiene cura; solo se pueden paliar sus síntomas para intentar mejorar la calidad de vida de las personas afectadas desde un punto de vista multidisciplinar (CalzadaSierra y Gómez-Fernández, 2001).

En cuanto a su origen, este se desconoce en un $90 \%-95 \%$ de los casos (ELA esporádica); el resto es de tipo hereditario (ELA familiar), entre un $5 \%$ y un $10 \%$ (Fundación Luzón, 2017). Al respecto, cabe destacar que los casos de ELA con origen hereditario presentan un inicio a edades más tempranas -diez años antes en muchas ocasiones- (Ministerio de Sanidad, Servicios Sociales e Igualdad, 2009).

La Asociación Valenciana de Esclerosis Lateral Amiotrófica (ADELA-CV) destaca este mismo aspecto: el desconocimiento sobre su origen. Sin embargo, pone el énfasis en la confluencia de diversos mecanismos alterados (ADELA-CV, 2017):

- Alteraciones en el metabolismo del glutamato (aminoácido esencial para la conducción del impulso nervioso.

- Sensibilidad aumentada al daño neuronal por productos tóxicos del metabolismo (daño hidroxiradical).

- Reducción de los factores del crecimiento neuronal.

- Muerte prematura de las neuronas por un mecanismo de "suicidio celular".

- Factores genéticos: defectos en la conformación normas de las cadenas de ADN que, en ocasiones, puede transmitirse por herencias. 
Según la Junta de Andalucía (2012), entre su sintomatología se encuentra la pérdida de destreza y fuerza muscular (debilidad), el aumento del tono o espasticidad, la exaltación de reflejos de estiramiento muscular o hiperreflexia patológica, los reflejos patológicos, la exaltación de reflejos en una extremidad atrófica, la parálisis pseudobulbar (espástica) y la labilidad emocional. Sin embargo, los estadios iniciales de la ELA no responden a un patrón clínico único y definido, sino que se caracterizan por una gran variabilidad clínica. Así, esta enfermedad puede comenzar indistintamente en los músculos del habla, en la función de tragar o en las manos, brazos, piernas o pies. Por ello, muchos de estos síntomas suelen confundirse con los de otras enfermedades. Este hecho y la ausencia de biomarcadores biológicos complican su diagnóstico precoz (CaballeroHernández et al., 2015).

En cualquier caso, la ELA afecta también gravemente al entorno familiar y a las y los profesionales encargados de llevar a la práctica los cuidados paliativos: fisioterapeutas, terapeutas ocupacionales, logopedas, psicólogos y psicólogas, personal de enfermería especializado, personal técnico, trabajadores y trabajadoras sociales, y personal médico rehabilitador. Por ello, en esta investigación cualitativa se han tenido en cuenta las vivencias de todas estas personas.

La siguiente tabla muestra qué profesionales intervienen cuando aparecen los síntomas antes citados; con la neurología como fuente aglutinadora de todos ellos.

Tabla 1. Síntomas de la ELA y profesionales implicados en los cuidados paliativos de la enfermedad.

\begin{tabular}{|l|l|}
\hline \multicolumn{2}{|c|}{ Proceso de la ELA y profesionales implicados en los cuidados paliativos } \\
\hline \multicolumn{1}{|c|}{ Síntomas } & \multicolumn{1}{c|}{ Profesionales implicados } \\
\hline $\begin{array}{l}\text { Disfagia, disartria, sialorrea, problemas de comunicación, } \\
\text { problemas socioeconómicos, afagia, desnutrición, dislipemia, } \\
\text { depresión, debilidad, hombro doloroso, espasticidad, disnea, } \\
\text { secreciones, debilidad, etc. }\end{array}$ & $\begin{array}{l}\text { Foniatra, logopedia, fisioterapia, terapia ocupacional, } \\
\text { psicología, PEG endoscópica o percutánea, asesor/a de } \\
\text { productos de apoyo, nutrición, trabajo social, etc. }\end{array}$ \\
\hline
\end{tabular}

Fuente: Elaboración propia

Como se puede observar en la tabla, entre los síntomas de esta enfermedad no se encuentran la disminución de las capacidad mental: la ELA no afecta a la inteligencia, el juicio, la memoria o los sentidos, aunque puede incidir negativamente en las emociones de las personas afectadas por esta enfermedad, en su entorno y en las y los profesionales que los y las atienden (Visús Susín, 2013). En el siguiente apartado se desarrollarán estos aspectos.
En cuanto a su prevalencia a escala internacional, según Camacho, Esteban y Paradas (2014) la ELA es la tercera enfermedad neurodegenerativa tras la demencia y el Parkinson. En general, indican estos autores, la incidencia en Occidente oscila entre uno y dos casos por cada cien mil habitantes al año. Por su parte, el número de personas que vive con ELA en la Comunidad Valenciana se aproxima a cuatrocientas y la incidencia de esta enfermedad en la sociedad española es de $2 / 100.000$, por lo que el riesgo de sufrirla 
es de uno entre mil. Se estima que cuarenta mil españoles y españolas sanos en la actualidad desarrollarán ELA, probablemente debido a la mayor esperanza de vida. En la actualidad, se estima que en España existen unas cuatro mil personas afectadas (ELA Principado, 2017).

Según el Instituto Nacional de Estadísticas (INE), en 2011 murieron en España 965 pacientes con ELA, prácticamente el mismo número de los nuevos casos diagnosticados ese mismo año. Sin embargo, el número de defunciones ha crecido desde 2000, año el que murieron 682 personas por esta causa. Este hecho puede deberse a una mejor codificación y conocimiento de la enfermedad (Ministerio de Sanidad, Servicios Sociales e Igualdad, 2016).

Otra cuestión importante es la edad media de inicio de la ELA: entre los sesenta y los 69 años, con un pico de incidencia a los setenta a 75 años y una disminución en edades superiores, a diferencia de lo que ocurre con la enfermedad del Parkinson o el Alzheimer (Camacho, Esteban y Paradas, 2015).

Asimismo, la ELA es incurable y mortal en un breve plazo de tiempo; la supervivencia media es de dos a cinco años (Fundación Luzón, 2017).

\section{Efectos de la ELA en las y los profesionales sociosanitarios}

Como ya se ha comentado, la ausencia de un tratamiento adecuado y multidisciplinar para la ELA provoca una actitud negativa en las personas afectadas por la enfermedad, sus familias y las y los profesionales que les atienden. En este sentido, las y los profesionales sociosanitarios encargados de las personas con enfermedades terminales como la ELA son un colectivo especialmente expuesto a factores de riesgo psicosociales (burnout, desgaste por empatía y depresión, entre otros), lo que afecta a su calidad de vida.

Al respecto, Fligey (1997) señala que el burnout y el desgaste por empatía están relacionados con la vulnerabilidad emocional sostenida, por lo que a continuación se desarrollarán estos conceptos someramente.

El burnout fue descrito por primera vez en 1974 por Freudenberger para referirse al estado de agotamiento físico y mental que observó entre las y los voluntarios de una clínica de desintoxicación. Lo definió entonces como "un quemarse en contraste con el 'ardiente entusiasmo' del principio".

El otro concepto estrechamente relacionado con la vulnerabilidad de las y los profesionales sociosanitarios que atienden a personas en el final de sus vidas es la fatiga por compasión, expresada de distintas formas (Figley, 1995 y 1997; Stamm, 1995, 1997; Mc. Cann y Pearlman, 1995; Pearlman y Saakvitne, 1995).

No obstante, todos estos autores y autoras se refieren a la fatiga por compasión que padecen las y los profesionales que trabajan con el sufrimiento humano. Este concepto se define como un tipo de estrés resultante de la relación de ayuda terapéutica, de la empatía y del compromiso emocional con las y los pacientes que sufren y padecen (Myezyentseva, 2014).

Algunos investigadores e investigadoras (O'Kearney y Perrott 2006) equiparan la fatiga por compasión con el trastorno por estrés postraumático (TEPT). Según ellos, los que sienten y expresan empatía más intensamente tienen también un mayor riesgo de internalizar el trauma. 
Sin embargo, en contraste con el burnout, los profesionales con fatiga por compasión pueden seguir ejerciendo sus funciones de forma comprometida (Garfield, 1994), aunque Fligey (2002) advierte de que la fatiga por compasión puede desencadenar el burnout.

En concreto y en lo que se refiere a la práctica de enfermería, Van Bogaert, Van Heusden, Timmermans y Franck (2014) señalan que el entorno en el que trabajan, caracterizado por diversas dificultades, favorece la aparición de factores de estrés que pueden "socavar la capacidad total del personal de enfermería para proporcionar una excelente atención".

Autores como Izquierdo (2012) cifran el problema en Europa de la siguiente forma: el $9 \%$ de las y los trabajadores ha sufrido mobbing, un $20 \%$ burnout y un $22 \%$ algún tipo de estrés laboral. No se conocen datos específicos que abarquen a la totalidad de las y los profesionales de este estudio, pero cabe suponer que en su caso, y por lo dicho anteriormente, las cifras se disparen. Al respecto, López-Soriano y Bernal (2002), en un estudio realizado a 223 trabajadores y trabajadoras dependientes de la dirección de enfermería de un hospital comarcal de Murcia, detectaron niveles preocupantes de burnout. En concreto, el 26,5\% presentó puntuaciones elevadas de agotamiento emocional y el $30 \%$ de despersonalización. A su vez, un 20,2\% puntuó bajo en falta de realización personal.

Por su parte, Frankl (1984) aporta una visión más positiva cuando invita a las y los profesionales a repensarse como personas $y$, además, como "especialistas en personas". Stamm (2002) identifica esta nueva concepción como un factor que contrarresta la fatiga por compasión y sugiere que este hecho podría explicar la existencia de la resiliencia humana, término muy relacionado con la satisfacción de la compasión (Calhoun, Cann, Tedeshi y McMillan, 1998).

\section{Estrategias de afrontamiento}

El término "afrontamiento" fue desarrollado a partir de los años ochenta por autores como Phillips (1984) para tratar de explicar las formas que emplea el ser humano para afrontar experiencias vitales estresantes. En los años setenta, el sociólogo Aaron Antonovsky ya había planteado en sus investigaciones lo nocivo que podía resultar el estrés y la necesidad de desarrollar programas de salud y calidad de vida para contrarrestar sus efectos.

Históricamente las investigaciones se han centrado en los factores psicosociales negativos que provocan situaciones estresantes como es estar en contacto con la enfermedad o la muerte (Contreras y Esguerra, 2006). Sin embargo, con la aparición a finales del siglo XX de la psicología positiva ${ }^{2}$, Seligman y Csikszentmihalyi (2000), en la actualidad se plantean el caso contrario para intentar analizar los mecanismos que repercuten y benefician positivamente a las personas para poder superar estas situaciones.

Seligman (2005) señaló la importancia de trabajar sobre las fortalezas de que dispone cada individuo. Las fortalezas son rasgos o características psicológicas que se presentan en situaciones distintas a través del tiempo, y sus consecuencias suelen ser positivas. Así, señala el autor, poner en práctica las fortalezas que cada uno posee provoca emociones positivas auténticas y actúa como barrera contra la enfermedad. Entre estas fortalezas destacan las siguientes: el optimismo, las habilidades interpersonales, la fe, el

\footnotetext{
${ }^{2}$ La psicología positiva es el estudio científico de las experiencias positivas, los rasgos individuales positivos, las instituciones que facilitan su desarrollo y los programas que ayudan a mejorar la calidad de vida de los individuos, mientras previene o reduce la incidencia de una psicopatología determinada (Seligman, 2005; Seligman y Csikszentmihalyi, 2000).
} 
trabajo ético, la esperanza, la honestidad, la perseverancia y la capacidad para fluir (flow) (Seligman y Christopher, 2000).

Otros autores (McNulty y Fincham, 2012), sin embargo, insisten en la necesidad de avanzar en el enfoque de la psicología positiva, ampliando el horizonte para estudiar los factores que determinan cuándo, por quién y en qué medida estos factores señalados están asociados con el bienestar.

Por su parte, Poseck (2006) coincide con los autores antes mencionados y señala que el énfasis e interés de la psicología clásica de centrarse en la enfermedad y sus aspectos negativos le ha hecho descuidar emociones positivas tales como el bienestar, la esperanza, el humor, la creatividad, la satisfacción, el optimismo y la felicidad.

No obstante, desde el punto de vista que nos ocupa -las y los profesionales sociosanitarios encargados de cuidar a las personas afectadas por la ELA- es importante destacar lo que Siegrist (1998) definió como "las recompensas y las satisfacciones laborales". Este autor pergeñó uno de los principales modelos para analizar el papel moderador de las recompensas y señaló que la falta de equilibrio entre "esfuerzos" y "recompensas" genera un estado de distrés que puede llevar a situaciones de tensión.

Así, ambas corrientes destacan la importancia de la interacción entre las situaciones externas y las personales; la situación organizacional y las fortalezas. En este sentido, y de acuerdo también con Torestad, Magnuson y Oláh (1990), el afrontamiento está determinado tanto por la situación como por la persona. Fernández-Abascal y Palmero (1999), por su parte, indican que las estrategias de afrontamiento son un conjunto de esfuerzos cognitivos y conductuales, constantemente cambiantes, que se desarrollan para manejar demandas específicas, externas e internas, que el individuo evalúa como desbordantes. Así pues, las estrategias son múltiples ya que el ser humano maneja distintas formas de afrontar situaciones diversas, y eso se relaciona con las creencias, los valores, la habilidades para interactuar y las capacidades para enfrentarse a situaciones difíciles, tal y como se verá en el apartado dedicado a exponer los resultados.

Otros autores abordan las diversas variables que afectan a las personas dedicadas al cuidado a la hora de afrontar su trabajo. Vachon (1987), por ejemplo, señala la juventud y escasez de habilidades de afrontamiento como un factor determinante en la aparición del síndrome de burnout. Otros investigadores especializados en cuidados paliativos (Escot, Artero, Gandubert, Boulenger y Ritchie, 2001) describen variables moduladoras como el sexo (los varones son más proclives a la despersonalización), la edad (se desarrollan estrategias de afrontamiento eficaces y niveladas), el estado civil (las personas casadas experimentan menos desgaste por el apoyo emocional que reciben), la antigüedad en el puesto de trabajo y la profesión. Sin embargo, todas estas variables deben ser analizadas en su conjunto, en un individuo concreto y en el marco de unas circunstancias específicas.

En cuanto a las diferencias de profesión a las que aludían los anteriores autores, parece que los y las enfermeras tienen mayores niveles de desgaste que las y los médicos (Martín Montero et al., 2000) porque están en contacto más continuo, aunque otros autores (Flórez, 2000) indican lo contrario.

Sin embargo, frente a las cuestiones antes enumeradas que colocan las y los profesionales sociosanitarios en una 
situación de vulnerabilidad, existen ciertas actuaciones que pueden reforzar su posición. Al respecto, Arranz, Barbero, Barreto y Bayés (2008) proponen las siguientes cuestiones para mejorar la actuación de estas y estos:

1. Formación: les dota de recursos para hacer frente a las dificultades, sobre todo en relación con el manejo de habilidades y control de emociones.

2. Cultura del trabajo en equipo con objetivos explícitos y realistas.

3. Realizar actividades gratificantes fuera del entorno laboral.

4. Fomentar la actitud de disfrute y de sentido del humor.

5. Saber pedir ayuda. Admitir los propios límites (Sandrin, 2005).

6. Compartir información y dificultades consensuando las decisiones.

Pearlman y MacCann (1995) añaden a todos estos aspectos el de dar y recibir supervisión porque, según el autor, este hecho alivia la carga emocional sufrida por el o la profesional y le ayuda a reconectarse con la realidad. El autor señala otros instrumentos para lidiar con el malestar laboral (el humor, la psicoeducación y la formación, y el desarrollo de técnicas de autocuidado).

\section{Objetivos}

El objetivo principal es indagar sobre cuáles son las percepciones del afrontamiento de la ELA que tienen las y los profesionales sociosanitarios que trabajan con personas afectadas por esta enfermedad. En concreto, los objetivos específicos son los siguientes:

a) Conocer qué estrategias utilizan las y los profesionales sociosanitarios para afrontar la intervención con personas afectadas de ELA.

b) Averiguar qué ámbitos abarcan en su intervención. c) Conocer cómo es la formación que tienen en comunicación de malas noticias, afrontamiento de la enfermedad y duelo, y cómo la mejorarían.

d) Conocer la satisfacción profesional que les produce su intervención.

\section{Metodología}

\section{Diseño de la investigación}

Para explorar las percepciones de afrontamiento de la ELA del personal sociosanitario se planificó, desde una perspectiva cualitativa, un análisis del contenido de las entrevistas semiestructuradas realizadas a una muestra de profesionales de distintas especialidades de la provincia de Valencia que tenían contacto directo con las personas afectadas por la enfermedad. La principal ventaja que presentan las entrevistas semiestructuradas, como señala Ander-Egg (1982), es que permiten profundizar en temas concretos y conocer de forma holística un área proporcionando una descripción más amplia de la que se obtiene con otros instrumentos. Se ha considerado que la metodología cualitativa era la más adecuada a la finalidad de la investigación en cuanto que privilegia la dimensión subjetiva, es decir, las maneras en que los individuos representan la realidad social (López, 2005).

\section{Participantes}

Previamente a la selección de la muestra para la investigación, entre diciembre de 2013 y enero de 2014 se había realizado un estudio de campo con el objetivo de conocer el contexto de los afectados de ELA. Se quería concretar a qué tipo de profesionales había que entrevistar en el estudio de percepción, cuáles debían ser los criterios de inclusión o exclusión de las y los participantes y qué debía contener el instrumento que se iba a utilizar. Para ello se entrevistó a ocho profesionales que estaban en contacto directo con las 
y los afectados de ELA: la presidenta y un miembro de la junta directiva de la asociación ADELA-CV, un psicólogo, una trabajadora social, un fisioterapeuta, un técnico sociosanitario, una terapeuta ocupacional y un experto en productos de apoyo. Se concertó una cita con cada una de estas personas y se les aplicó un borrador de entrevista.

Tras analizar los resultados del estudio de campo, se decidió que en la investigación se quería contar con profesionales del área social, del área de la salud y también de algunas otras áreas colindantes que tratan a las personas afectadas por la ELA. Para seleccionar a las y los participantes se recurrió a ADELA-CV, a cuatro hospitales públicos de la provincia de Valencia ( $H$. General Universitario, $\mathrm{H}$. Universitario y Politécnico La Fe, $\mathrm{H}$. Clínico Universitario y $\mathrm{H}$. de Requena), a la Universidad Católica de Valencia (UCV) y a la empresa BJ Adaptaciones; en cada una de estas entidades se contactó con profesionales de distintas ramas que mantenían relación directa con personas afectadas por la ELA (véase Figura 1).

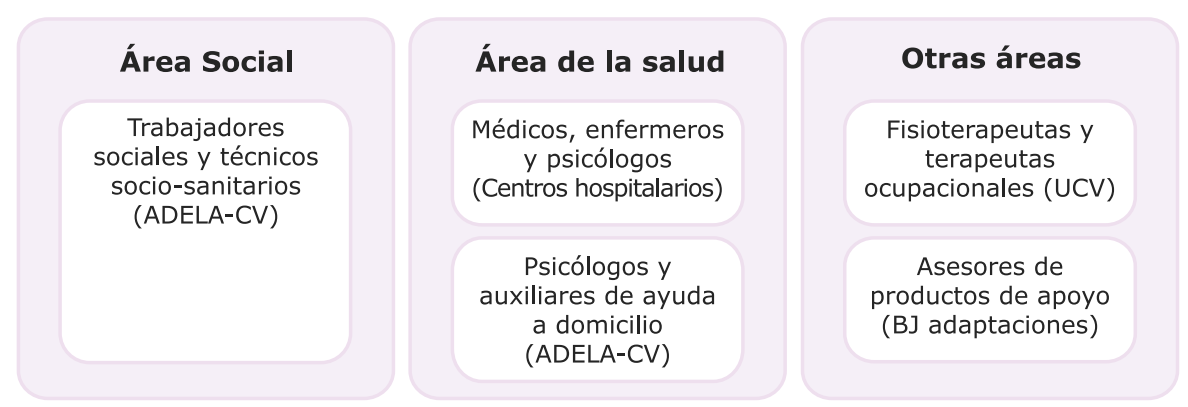

Figura 1. Áreas abarcadas en el estudio

Se determinaron como criterios de inclusión de las y los participantes que fueran profesionales asalariados que estuvieran trabajando con personas afectadas por la ELA desde, al menos, un mes antes a la entrevista, y que mantuvieran contacto directo con las personas afectadas 0 sus familias. Asimismo, como criterio de exclusión se estableció que no podían participar profesionales que trabajaran como voluntarios ni los que estuvieran afectados por la enfermedad (directamente o por un familiar).

Se estableció contacto con las entidades escogidas para solicitarles acceso a sus trabajadores. A través de correo electrónico se les explicó la investigación proyectada y se les solicitó su participación. Con quienes quisieron colaborar se fijaron citas individuales en sus centros de trabajo para poder recoger la información en un ambiente en el que se sintieran cómodas y cómodos y se les solicitó permiso para grabar las entrevistas. La participación ha sido desinteresada y se ha garantizado la confidencialidad de los datos que nos proporcionaron. Las entrevistas se realizaron entre el 4 de marzo y el 28 de mayo de 2014.

Finalmente, se ha contado con veintidós profesionales (el $59,1 \%$ son mujeres y el $40,9 \%$ hombres) con una media de edad de 44 años. El $72,7 \%$ de las y los participantes tenía pareja en el momento de la entrevista y un $63,6 \%$ tenía hijos o hijas. 
El $68,2 \%$ de las y los profesionales trabajaban en el área de la salud, el $13,6 \%$ en el área social y el $18,2 \%$ en otras áreas (Figura 2). Respecto a su centro de trabajo, la mitad procedía de hospitales públicos, el $31,8 \%$ de ADELACV, el $13,6 \%$ de la UCV y el $4,5 \%$ eran asesores de productos de apoyo de la empresa de adaptaciones BJ (Figura 3).

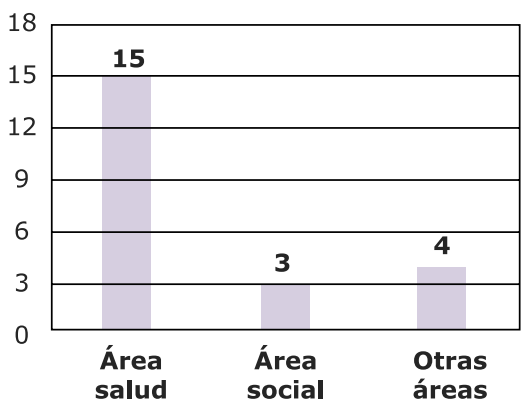

Figura 2. Áreas de trabajo de las y los participantes

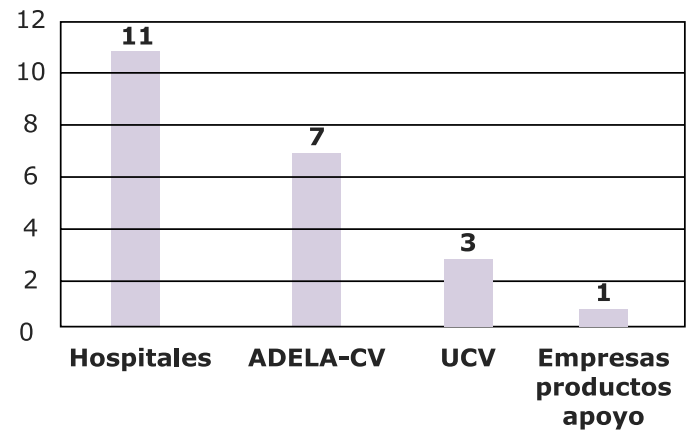

Figura 3. Lugar de trabajo de las y los participantes

en concreto, tratando a pacientes de ELA, una media de diez años de experiencia $(\mathrm{DT}=8,12)$.

\section{Instrumentos}

Tras analizar las entrevistas del estudio de campo, se diseñó un cuestionario que se administró en una prueba piloto a tres profesionales de atención sociosanitaria para comprobar que era adecuado para la investigación. El instrumento definitivo fue un cuestionario semiestructurado de carácter cuali-cuantitativo, con preguntas abiertas y cerradas, que incluía veintidós ítems relativos a los siguientes aspectos:

a) Características sociodemográficas y laborales de la o el entrevistado (ocho ítems): relativas al sexo, fecha de nacimiento, pareja, hijos e hijas, profesión y experiencia.

b) Estrategias de afrontamiento de las y los entrevistados con las personas afectadas de ELA y su autoevaluación del afrontamiento racional y emocional (cuatro ítems).

c) Estrategias de afrontamiento en el proceso de duelo con la ELA y su media de quince años de práctica en el ejercicio de su profesión $(D T=11,27)$ y, 
autoevaluación del afrontamiento racional y emocional (cuatro ítems).

d) Formación que tienen y la que consideran que requieren para tratar con las y los afectados por la ELA (tres ítems).

e) Otros aspectos relativos a cómo intervienen las y los participantes con las personas afectadas por ELA y su nivel de bienestar al ejercer su profesión (dos ítems).

f) Autoevaluación del nivel de tensión al realizar la entrevista (un ítem).

La cumplimentación del cuestionario tenía una duración estimada de diez a quince minutos.

\section{Análisis de datos}

A partir de las grabaciones de las entrevistas se transcribió lo comunicado por las y los participantes tanto de forma verbal como no verbal.

Para abordar analíticamente el objetivo principal de la investigación -las estrategias de afrontamientodos investigadoras estudiaron las transcripciones de forma independiente. En ellas identificaron las unidades de significado relevantes y les asignaron códigos. Posteriormente contrastaron las categorías que había establecido cada una, manteniendo la mayoría de ellas al ser coincidentes y fáciles de inferir, y modificando algunas otras. A continuación, ambas investigadoras procedieron a analizar las entrevistas clasificando la información de acuerdo a la categorización previa, controlando después si los resultados de ambas eran coincidentes y llegando a un consenso en los casos discrepantes.

\section{Resultados}

\section{Estrategias de afrontamiento}

\section{Aceptación}

Una de las estrategias que se han evidenciado en las personas entrevistadas es la aceptación de la situación: "Me planteo la enfermedad como una enfermedad más [...] es el juego de la vida y nosotros estamos jugando a ese juego de la vida" (trabajadora social); "forma parte de la medicina el asumir que los pacientes se te mueren" (neurólogo); "siempre cuesta de decir, pero, al mismo tiempo, sabes que lo tienes que hacer" (neumóloga); "pienso que la muerte es inherente a la vida y que la enfermedad ocurre aunque hayamos hecho las cosas bien y no nos lo merezcamos" (foniatra).

También la aceptación se produce, pero desde otro planteamiento, como aceptación de las emociones que sienten durante la intervención: "Al principio te llevas historias a casa, lo que sería un factor de riesgo para el quemazón profesional, pero con el tiempo aprendes a autorregular las emociones; es decir, hay que reconocer que hay situaciones que a los profesionales nos dan miedo y para autorregularnos lo primero es saber las emociones que estas sintiendo y no negarlas, y luego compartirlas" (psicólogo); "en el duelo a quien ayudamos es a los familiares [...] y si tengo que llorar con ellos pues lloro y no pasa nada, porque yo tengo que hacer mi duelo" (enfermera).

\section{Naturalización}

Otra de las estrategias más habituales es la de naturalizar las propias emociones: "naturalizar mucho las emociones negativas y la muerte [...] aunque tenemos nuestros propios duelos" (psicólogo). Y naturalizar la enfermedad: "mi forma de afrontar una persona con ELA no es diferente a mi forma de afrontar con una 
persona que es paliativa" (psicólogo); "el duelo lo vivo con naturalidad en el sentido de que conozco perfectamente cómo es la evolución de la enfermedad" (neurólogo); "afronto mi propia muerte sin ningún miedo $y$, evidentemente, también la del paciente" (foniatra).

\section{Buena comunicación y confianza}

Otra estrategia es la aproximación a las personas afectadas: "lo que buscamos es trabajar de una forma en la que se busque siempre al paciente [...] siempre a nivel muy familiar" (fisioterapeuta); "Ios primeros contactos son bastante duros hasta que conoces a la persona y a todo su entorno familiar y te vas adaptando" (auxiliar a domicilio); "intentamos establecer una relación de confianza, que sepan que les vamos a ofrecer soluciones y no les vamos a presionar en exceso" (nutricionista); "tratas de saber exactamente qué es lo que saben de la enfermedad y lo que suponen para ser consciente de hasta qué punto pueden prever lo que pueden necesitar a medio-largo plazo. Intento que sea una conversación en la que yo voy dirigiendo los temas y ellos libremente me comentan [...] intento que el afectado se implique" (trabajadora social).

\section{Consultas a compañeros y compañeras}

También hay profesionales que tratan de compartir las responsabilidades para afrontar con mayor tranquilidad la enfermedad: "hemos intentado crear una especie de plan de actuación en la que estén incluidos el neurólogo, el internista [...] de manera que las decisiones son más consensuadas, y eso te alivia y te quita mucha ansiedad" (médico de familia).

\section{Recompensa}

Algunas personas entrevistadas evalúan los aspectos positivos y los negativos $y$ obtienen un salario emocional positivo de su intervención: "hacer una compensación de qué te gratifica más, el saber que mínimamente contribuyes a su bienestar o el dolor que tú puedes tener [...] el poder ayudarles lo que se pueda siempre compensa más que lo malo" (psicólogo).

\section{Humor}

Otra estrategia que usan las y los profesionales en su ámbito personal para afrontar la enfermedad cuando no están con las y los enfermos es a través del humor: "de alguna manera aprendes a hacer un cierto grado de separación muchas veces a través del humor [...] para quitarles peso a todas las emociones que has podido sentir en la consulta" (neurólogo).

Sentir que se hace un buen trabajo

Algunas personas entrevistadas lo que utilizan es valorar la ayuda que dan a las y los pacientes: "me resulta, no digo agradable, pero sí gratificante el poder acompañar, desde el primer momento en que se le comunica hasta el final, a mi paciente, porque ahora mi objetivo es que tenga la mejor calidad de vida, pero también darle la mejor calidad de muerte [...] me resulta reconfortante saber que conmigo pueden contar" (foniatra); "siempre me dirijo a las personas afectadas con ELA de una forma positiva, pensando siempre en sus capacidades y no en sus limitaciones y en potenciar al máximo esas capacidades" (asesora de apoyo); "cuando veo de forma progresiva la enfermedad, me hace ser quizás un poquito más fuerte y ser mejor profesional, porque yo creo que nuestra misión es mucha de acompañamiento, asesoramiento [...] y de ayudar" (terapeuta ocupacional).

\section{Empatía}

A algunos y algunas profesionales les sirve buscar en el contacto el entendimiento de los sentimientos y emociones de la persona con ELA y su familia: "lo compartes más con los 
propios pacientes [...] hace más dura la experiencia de la enfermedad porque lo vives más intensamente, pero también lo dotas de más significado" (neuróloga); "no puedes decaer porque si te ven a ti decaer, decaen ellos" (trabajadora social); "me implico hasta que llega un momento en que cuando un paciente está muy mal hay un nivel en el que se estabiliza porque quizás las pérdidas ya no se notan tanto y lo que quiere es liberarse, entonces a mí eso me permite bajar un poco el nivel" (foniatra).

\section{Separación}

Otra estrategia de afrontamiento es la de establecer distancias para afrontar la situación: "me pongo límites, barreras" (trabajadora social); "intentamos no vincularnos mucho a nivel personal, si no, no podríamos ayudarles" (fisioterapeuta); "al principio, desde luego, es duro, lo que pasa es que a medida que vas conociendo afectados intentas que te afecte de la menor forma posible" (auxiliar a domicilio); "te cuesta, pero es que es lo que hay, yo creo que se marca una cierta distancia emocional, aunque seas cercano al paciente, si no, no le puedes ayudar" (foniatra); "trato de tener un poquito de límites sabiendo la situación y conociendo la evolución de esta enfermedad" (enfermera).

\section{Negación}

Hay profesionales a quienes les cuesta aceptar que la enfermedad que tienen que diagnosticar es la ELA y a veces tratan de negar su existencia: "No te gusta diagnosticar esta enfermedad, intentas demostrar que es otra cosa a sabiendas de que muchas veces el primer día que ves al enfermo tienes seguro que es una ELA" (neurólogo).

\section{Ámbitos que se abarcan en el tratamiento}

Las y los profesionales que tratan con las personas afectadas por la ELA y que no tienen atribuidas entre sus competencias ocuparse de los aspectos emocionales, han explicado que, en el caso de esta enfermedad, realizar su trabajo incluye, no solo dar asesoramiento e información, sino también procurar a las y los enfermos soporte emocional. Explican que estas personas y sus familiares necesitan ser escuchados y entendidos porque si los tratan así, eso les ayuda mucho a ellas y ellos mismos en el proceso. Consideran, por tanto, que en esta enfermedad, para dar una buena atención a las personas afectadas, su trabajo requiere más implicación personal que en otras; en consecuencia, las estrategias de afrontamiento que requieren ante la ELA pueden ser distintas a las que desarrollan al afrontar otras dolencias.

En el caso concreto de los psicólogos, se pone de manifiesto que sus actuaciones con las personas afectadas por la ELA son muy amplias, porque se encargan de informar, orientar, dar apoyo psicológico, ayudar en la adaptación y tratan no solo con las personas con ELA sino también con sus familias. Consideran que en muchos casos hacen counseling y desarrollo de protocolos de intervención psicológica para esta enfermedad.

\section{Formación en comunicación con pacientes, comunicación de malas noticias, afrontamiento y duelo}

Las personas entrevistadas nos han explicado que algunas y algunos pacientes afectados por la ELA y sus familiares se quejan de cómo ha sido el trato recibido por parte de las y los profesionales que les atienden y de la forma en que les han dado el diagnóstico de la enfermedad, argumentando que esas formas inadecuadas les han acarreado más miedos y frustraciones. También dicen que algunas $y$ algunos profesionales tienen dificultades en el afrontamiento de la ELA, ya que se bloquean en las consultas cuando están con esta clase de 
pacientes y no tienen las herramientas necesarias para su trato.

Al analizar las entrevistas, hemos comprobado que casi todas las personas consideran que es insuficiente la formación que tienen en estos temas $y$, en la mayoría de los casos, quienes dicen tener formación en comunicación de malas noticias, acompañamiento en la enfermedad y afrontamiento del duelo la han adquirido motu proprio, es decir, no se la han proporcionado en sus centros de trabajo para que puedan desempeñar mejor su labor ni tampoco se la han requerido. Nos han explicado que esa formación adicional a sus estudios la han obtenido por varias vías: másteres o cursos que han realizado a posteriori, lectura de libros, etc. Solo, excepcionalmente, un médico dijo que en su unidad se daban talleres sobre estos temas.

Otras y otros profesionales indican que, aunque no tienen formación, la práctica diaria es lo que les ha servido para realizar mejor estas tareas, y se quejan de que, mientras que para otras enfermedades como el cáncer sí que se proporciona este tipo de formación, para la ELA no la hay.

Por el contrario, cabe señalar que algunas personas entrevistadas explican que hay colegas a quienes estas cuestiones no les interesan y que no trabajan nunca con las emociones; también hay argumentos que justifican que la rotación de personal en los hospitales es lo que hace que haya trabajadores y trabajadoras que no adquieran experiencia con este tipo de enfermedades y no sepan cómo intervenir correctamente en el plano emocional.

En general, las personas entrevistadas piensan que necesitan más formación para tratar con este tipo de pacientes y que las mejoras deberían encaminarse hacia los siguientes aspectos:
- Saber gestionar sus propias emociones.

- Introducir casos prácticos durante sus estudios.

- Psicoeducación para evitar el burnout y la fatiga de compasión.

- Técnicas para favorecer el contacto directo con pacientes.

- Formación en counseling.

- Conocer todos los aspectos de la ELA.

- Aumentar la sensibilidad de las y los profesionales con los pacientes con una enfermedad, especialmente cuando esta es terminal.

- Aportar los recursos necesarios a las y los profesionales para que se sientan fuertes y bien capacitados para atender a las personas afectadas por esta enfermedad y a sus familias.

- Fomentar la asertividad con las personas afectadas por este tipo de enfermedades.

En lo relativo a cuándo tiene que recibirse la formación, piden que sea durante la carrera universitaria o durante la jornada laboral y señalan que debería incluirse en los centros de atención primaria porque son los más cercanos a las y los pacientes, donde son vistos día a día y a cuyos profesionales se les plantean muchas dudas. Además, consideran que sería positivo contar en este tipo de formación con profesionales que tengan experiencia con personas afectadas por esta enfermedad o con otras enfermedades que cursen con síntomas semejantes.

\section{Satisfacción profesional}

Aunque las personas entrevistadas recurren a estrategias distintas para hacer frente a la ELA, en general, son profesionales que están satisfechos y satisfechas con su trabajo con las personas afectadas. Dicen que les satisface el bienestar que pueden transmitirles a estas personas y a sus familiares; saber que, aunque la situación sea muy difícil, han ayudado en lo que han podido. Así, 
la neumóloga señalaba que "ya que no puedes dar una buena vida, al menos puedas dar una buena muerte". Sin embargo, aunque tengan satisfacción en este sentido, no son conformistas; algunos de ellos y ellas señalan que les gustaría tener más experiencia con las personas con ELA para poder desarrollar mejor su trabajo.

Por el contrario, algunos y algunas profesionales valoran su satisfacción según las reacciones de las personas afectadas por la ELA y sus familias: si consiguen ayudarles están satisfechos, si no, se frustran. También argumentan que, aunque les gusta su profesión y ven cosas que les hacen pensar que están actuando bien, hay muchas acciones de las que no llegan a ver los resultados. Uno de los foniatras, por ejemplo, nos explicó que él no estaba muy satisfecho porque sentía que en realidad podía ayudarles poco.

\section{Conclusiones}

La ELA es una enfermedad que, por sí misma y por todos los problemas adicionales que lleva aparejada, puede generar dificultades de afrontamiento en las personas que la padecen y sus familiares. Pero, además, es importante señalar que también puede menoscabar a las demás personas que están en el entorno de las personas afectadas por la ELA, entre ellos, a las y los profesionales sociosanitarios que les atienden. Esta sensibilidad particular del entorno hacia la ELA se ha visto reflejada en el estudio al comprobar cómo un gran número de las y los profesionales entrevistados recordaban perfectamente a sus primeros y primeras pacientes, aunque hubieran transcurrido veinte años de haberlas y haberlos tratado.

Sin embargo, los efectos que esta enfermedad causa en las y los profesionales sociosanitarios están relacionados con las estrategias que desarrollan para afrontarla. En el estudio se refleja que las y los profesionales llevan a la práctica diversas estrategias para poder efectuar su afrontamiento personal de la enfermedad. Una de las más extendidas es la aceptación, tanto de la enfermedad como del reconocimiento de los propios sentimientos y emociones que les genera. Otra de las estrategias a la que más recurren es la de naturalizar la enfermedad que están tratando como uno más de los obstáculos que las personas pueden encontrar en la vida y la de naturalizar la muerte en que la enfermedad culmina como una etapa que es inherente a la propia vida.

Asimismo, las formas en que estas y estos profesionales afrontan la enfermedad en muchas ocasiones dependen del papel que desempeñan en su proceso. Así, para las y los profesionales encargados de transmitir el diagnóstico (neurólogos y neurólogas) a las y los enfermos, esta situación es dura y muy difícil, por lo que en ocasiones recurren a la negación de la realidad o al humor como estrategia de evasión. Quienes tratan a las y los pacientes en momentos en los que ya son conscientes de lo que tienen (fisioterapeutas, auxiliares a domicilio, etc.) buscan más una relación de confianza con las personas afectadas por la ELA e intentan separar los casos que atienden del resto de sus vidas. Las y los trabajadores que les dan herramientas para "facilitarles" su vida diaria (foniatras, asesores y asesoras de productos de apoyo, etc.) lo afrontan desde el pensamiento de que hacen un buen trabajo, porque les ayudan a convivir con su enfermedad.

Por otra parte, las y los profesionales consideran que, no solo tienen que asesorar e informar, sino que también tienen que dar a las personas afectadas $y$ 
a sus familias soporte emocional. En este punto es importante resaltar que sería recomendable que se les proporcionara formación para que, al saber afrontar mejor la enfermedad, también pudieran hacer su función con mayores garantías.

En esa misma línea, las y los profesionales creen que la formación que tienen en comunicación de malas noticias, afrontamiento y duelo es insuficiente $y$, si la tienen, es porque se han preocupado personalmente en adquirirla. Por ello reclaman más formación práctica que les ayude a realizar su trabajo, porque redundará en su propio beneficio pero también en el de las personas que atienden.

En cuanto a su satisfacción profesional, aunque la ELA sea una enfermedad en ocasiones difícil de afrontar, asumen que no pueden salvar la vida a las personas afectadas, pero están satisfechos y satisfechas de poder trabajar con dichas personas y con sus familiares.

En definitiva, el estudio refleja que, aunque sin duda en un plano distinto al que corresponde a las y los enfermos y sus familiares, también es necesario incluir al personal sociosanitario en el ámbito de las personas afectadas por la ELA, ya que "necesitan" desarrollar estrategias de afrontamiento porque en ellos y ellas también se produce una serie de emociones que tienen que poder gestionar de la mejor manera posible. Estas estrategias de afrontamiento son diversas y estarán condicionadas por su función con la persona afectada por la ELA. La aplicación de las estrategias de afrontamiento podría ser más efectiva para su bienestar si se les facilitara la formación de la que carecen en este ámbito, tal y como se hace con las y los profesionales que se ocupan de otras enfermedades graves o incurables.

\section{Referencias bibliográficas}

ADELA-CV. (2017). Estrategia en enfermedades neurodegenerativas del sistema nacional de salud. Recuperado de: ADELA-CV. Recuperado de: http://adela-cv.org/

Arranz, P.; Barbero, J.J.; Barreto, P. y Bayés, R. (2008). Intervención emocional en cuidados paliativos. Modelo y Protocolos ( $3^{a}$ ed.). Barcelona, España: Ariel.

Caballero-Hernández, D.; Toscano, M.G.; Cejudo-Guillén, M., García-Martín, M.L. y López S. (2015). The "Omics" of Amyotrophic Lateral Sclerosis. Trends in Molecular Medicine, 22(1), $1-15$.
Calhoun, L.G.; Cann, A.; Tedeschi, R.G. y McMillan, J. (1998). Traumatic events and generational differences in assumptions about a just world. Journal of Social Psychology, 138, 789-791.

Calzada-Sierra, D. y GómezFernánderz, L. (2001). Importancia del tratamiento rehabilitador multifactorial en la esclerosis lateral amiotrófica. Revista de Neurología, $32,423-426$.

Camacho, A.; Esteban, J. y Paradas, C. (2014). Informe de impacto social de la ELA y las enfermedades neuromusculares. Fundación Española de Enfermedades Neurológicas. Recuperado de http://www.fundaciondelcerebro.es/docs/INFORME_ELA.pdf 
Camacho, A.; Esteban, J. y Paradas, C. (2014). Informe de impacto social de la ELA y las enfermedades neuromusculares. Fundación Española de Enfermedades Neurológicas. Recuperado de: http://www.fundaciondelcerebro.es/ docs/INFORME_ELA.pdf

Contreras, F., y Esguerra, G. (2006). Psicología positiva: una nueva perspectiva en psicología. Diversitas: Perspectivas en Psicología, 2(2), 311-319. Recuperado de http:// www.scielo.org.co/pdf/dpp/v2n2/ v2n2a11.pdf.

ELA Principado (2017). Recuperado de https://www.ela-principado.es/.

Escot, C.; Artero, S.; Gandubert, C.; Boulenger, J.P. y Ritchie, K. (2001). Stress level in nursing staff working in oncology. Stress and health, 17, 273-279. doi: 10.1002/smi.907.

Fernández-Abascal, E.G.F. y Palmero, F. (1999). Emociones y salud. Barcelona, España: Ariel.

Figley, C. R. y Stamm, B. H. (1995). Cuestionario autoaplicado de fatiga de compasión y satisfacción.

Figley, C. R. (1995). Compassion Fatigue: Coping with SecundaryTraumatic Stress Disorder in Those Who Treat the Traumatized. Estados Unidos, Nueva York: Brunner/Mazel Publishers.

Figley, C.R. (1997). Examples of Compassion Fatigue/Burnout Syndrome. Institute on Crisis Management in Higher Education. Recuperado de: http://www.chaffey. edu/profdev/fsc/events/fa10/ ExampleCF.pdf.
Figley, C. R. (2002). Treating Compassion Fatigue. London, Reino Unido: Taylor and Francis, Ltd.

Flórez, L. (2000). La Psicología de la Salud y la Salud Pública: El papel de la comunidad científica (Resumen). $5^{\circ}$ Congreso Iberoamericano de Psicología de la Salud. Calidad de Vida en el Siglo XXI, 27.

Frankl, V. (1984). Psicoterapia y humanismo. ¿Tiene un sentido la vida? Madrid, España: Fondo de Cultura Económica.

Freudenberger, H.J. (1974). Staff Burnout. Journal of Social Issues, 30, 159-165.

Fundación Luzón. (2017). Fundación Luzón. Recuperado de: http:// ffluzon.org/

Izquierdo, F.M. (2012). Manual de riesgos psicosociales en el trabajo: Teoría y práctica-introducción. Recuperado de: http://www.psicologia-online. com/ebooks/riesgos/introduccion. shtml.

Junta de Andalucía. (2012). Guía asistencial de esclerosis lateral amiotrófica. Recuperado de: http://www.juntadeandalucia.es/ salud/sites/csalud/contenidos/ Informacion_General/c_3_c_6_ enfermedades_raras/guia_ asistencial_esclerosis

Lazarus R. y Folkman S. (1986). Assessing coping strategies: A theoretically based approach. Journal of Personality and social Psychology, 56, 267- 283.

Lazarus, R. y Folkman, S. (1986). Estrés $y$ procesos cognitivos. Barcelona, España: Editorial Martínez-Roca. 
López-Soriano, F. y Bernal, L. (2002). Prevalencia y factores asociados con el síndrome de burnout en enfermería de atención hospitalaria. Revista de calidad asistencial, 17(4), 201-205.

McNulty, J. K. y Fincham, F. D. (2012). Beyond positive psychology? Toward a contextual view of psychological processes and well-being. American Psychologist, 67(2), 101. doi: 10.1037/a0024572.

Ministerio de Sanidad, Servicios Sociales e Igualdad (2009). Guía para la atención de la esclerosis lateral amiotrófica (ELA) en España. Recuperado de: https:// www.msssi.gob.es/profesionales/ prestaciones Sanitarias/ publicaciones/docs/esclerosisLA.pdf

Montero, C.M., Lorente, R. L., González, C. F., García, E. G., Trillo, R. O., Pendón, A. C. y Puche, J. G. (2000). Estudio comparativo del síndrome de burnout en profesionales de oncología: incidencia y gravedad. Med Pal,Medicina Paliativa 7(3), 8593.

Myezyentseva O. (2014). Fatiga por compasión en los profesionales de enfermería. Revisión sistemática [Trabajo fin de grado]. TecnoCampus, Mataró, España. Recuperado de:

O'Kearney, R. y Perrott, K. (2006). Trauma narratives in posttraumatic stress disorder: A review. Journal of Traumatic Stress, 19, 81-93.

Pearlman, L. A. y Mac Ian, P. S. (1995). Vicarious traumatization: An empirical study of the effects of trauma work on trauma therapists. Professional Psychology: Research and Practice, 26(6), 558.

Pearlman, L. A. y Saakvitne, K. W. (1995).
Treating therapist with vicarious traumatization and secondary traumatic stress disorders. En C.R. Figley (Ed.). Compassion fatigue: Coping with secondary traumatic stress disorder in those who treat the traumatized, pp. 150-177. Nueva York, Estados Unidos: Brunner/ Mazel.

Pearlman, L.A. y Saakvitne, K.W. (1995). Treating therapist with vicarious traumatization and secondary traumatic stress disorders. En C.R. Figley (Ed.). Compassion fatigue: Coping with secondary traumatic stress disorder in those who treat the traumatized, pp. 150-177. Nueva York, Estados Unidos: Brunner/ Mazel.

Phillips, J. R. (1984). Faculty burnout. American Journal Nursing, 82, 15251526.

Poseck, B.V. (2006). Positive Psychology: a new way of understanding Psychology. Psicólogo, 27(1), 3-8.

Sandrin, L. (2005). Ayudar sin quemarse. Cómo superar el Burn-out en las profesiones de ayuda. España, Madrid: Ediciones San Pablo.

Seligman, M.E.P. y Christopher, P. (2000). Positive Clinical Psychology. Seligman, M.E.P. y Christopher, P. (2000). Positive Clinical Psychology. Recuperado de: http://ppc.sas. upenn.edu/.

Seligman, M.E.P., y Csikszentmihalyi, M. (2000). Positive psychology: An introduction. American Psychologist, 55(1), 5-14.

Siegrist, J. (1998). Adverse health effects of effort-reward imbalance at work. Theories of organizational stress, 190-204. 
Sociedad Española de Neurología (2017). Informe de la Fundación del Cerebro sobre el impacto social de la esclerosis lateral amiotrófica y las enfermedades neuromusculares. Neurología, 32(9.). Elsevier.

Stamm, B. H. (1997). Work-related secondary traumatic stress. PTSD Research Quarterly, 8(2).

Stamm, B. H. (2002) Medir la satisfacción compasión, así como fatiga: Historia del desarrollo de la compasión y la satisfacción del ensayi de fatiga. En Cr Frigey (Ed.D), Tratamiento de la fatiga compasión, 107-119). Nueva York, Estados Unidos: BrunnerRoutledge.

Törestad, B.; Magnusson, D.; Oláh, A. (1990). Coping, Control and experience of anxiety: An interactional perspective. Anxiety Research, 3:1-16.

Vachon, M.L.S. (1987). Occupational stress in the Care of the Critically III, the Dying and the Bereaved. Washington, D.C., Estados Unidos: Hemisphere Publishing Corp.
Van Bogaert, P.; Van Heusden, D.; Timmermans, O. y Franck, E. (2014). Nurse work engagement impacts job outcome and nurse-assessed quality of care: model testing with nurse practice environment and nurse work characteristics as predictors. Frontiers in Psychology, 5, 1. doi: 10.3389/ fpsyg.2014.01261. Recuperado de: http://www.ncbi.nlm.nih.gov/pmc/ articles/PMC4230203/

Visús Susín, D. (2013). Programa de atención a pacientes con Esclerosis Lateral Amiotrófica (ELA): información para el personal sanitario de los Centros de Atención Primaria, [tesis doctoral]. Universitat de Lleida, Lleida, España.

Visús Susín, D. (2013). Programa de atención a pacientes con Esclerosis Lateral Amiotrófica (ELA): información para el personal sanitario de los Centros de Atención Primaria, [tesis doctoral]. Universitat de Lleida, Lleida, España.

Wiley J. Garfield SL. (1994). Research on client variables in psychotherapy. En: Bergin AE , Garfield SL (Ed.), editors,. Handbook of psychotherapy and behavior change (190-228.). New York, Estados Unidos: John Wiley. Bergin Garfield SL. 